\title{
Stratified medicine in schizophrenia: how accurate would a test of drug response need to be to achieve cost-effective improvements in quality of life?
}

\author{
Huajie Jin ${ }^{1}$. Paul McCrone ${ }^{1}$. James H. MacCabe ${ }^{2}$ \\ Received: 22 November 2018 / Accepted: 19 August 2019 / Published online: 28 August 2019 \\ (c) The Author(s) 2019
}

\begin{abstract}
Objective Stratified medicine refers to the use of tests that predict treatment response to drive treatment decisions for individual patient. The pharmacoeconomic implications of this approach in schizophrenia are unknown. We aimed to assess the cost-effectiveness of a hypothetical stratified medicine algorithm (SMA) compared with treatment as usual (TAU), for patients with schizophrenia who failed a first-line antipsychotic.

Methods A decision analytic model with embedded Markov process was constructed, which simulated the health and cost outcomes for patients followed SMA or TAU over a lifetime horizon, from healthcare and social care perspective. In the basecase analysis, sensitivity and specificity of the stratifier were both set as $60 \%$. Extensive sensitivity analyses were conducted to test the impact of uncertainty around the value of important parameters. The primary outcome was the incremental cost per quality-adjusted life year (QALY) gained.

Results When both sensitivity and specificity of the stratified test were set at 60\%, SMA appeared to be the optimal strategy as it produces more QALYs and incurs lower costs than TAU. This is robust to all scenarios tested. At a willingness-to-pay threshold of $£ 20,000$ per QALY, the probability for SMA to be the optimal strategy is $82.4 \%$.

Conclusions Our results suggest that use of any stratifier with a sensitivity and specificity over $60 \%$ is very likely to be costeffective comparing to TAU, for psychotic patients who failed a first-line antipsychotic. This finding, however, should be interpreted with caution due to lack of evidence for clozapine as a second-line antipsychotic.
\end{abstract}

Keywords Schizophrenia $\cdot$ Stratified medicine $\cdot$ Health economics $\cdot$ Cost-effectiveness $\cdot$ Markov model

JEL Classification D61

Electronic supplementary material The online version of this article (https://doi.org/10.1007/s10198-019-01108-4) contains supplementary material, which is available to authorized users.

Huajie Jin

huajie.jin@kcl.ac.uk

1 King's Health Economics (KHE), Institute of Psychiatry, Psychology \& Neuroscience at King's College London, Box 024, The David Goldberg Centre, London SE5 8AF, UK

2 The Department of Psychosis Studies, Institute of Psychiatry, Psychology \& Neuroscience at King's College London, London SE5 8AF, UK

\section{Introduction}

Stratified medicine refers to the use of a stratifier (e.g. a biomarker or other predictive marker) to guide treatment choices. The mainstay of treatment for schizophrenia is antipsychotic medication, however, about one-third of patients with schizophrenia are treatment resistant (TRS) - they do not respond adequately to conventional antipsychotics other than clozapine [1]. Clozapine is the only drug with established efficacy in reducing symptoms and the risk of relapse for adults with TRS [2]. In addition, clozapine is also superior for negative and cognitive symptoms, as well as reducing suicidality and all-cause mortality [3]. Owing to safety concerns regarding the risk of neutropenia and agranulocytosis, the use of clozapine in most countries is restricted to patients 
who fail two non-clozapine antipsychotics at adequate doses [2, 4-6]. Although theoretically patients could be trialled on two non-clozapine antipsychotics in the space of a few months, in practice there is frequently a delay of several years before clozapine is prescribed [7]. There is emerging evidence, that ineffective antipsychotic treatment early in disease leads to worse long-term outcomes [8], and specifically that delay in prescribing clozapine to patients who are treatment resistant confers a poor outcome [9]. Thus, a stratifier which could predict TRS accurately and early might allow better decision making and lead to improved patients' outcomes.

Significant efforts have been made to identify different stratifiers for predicting antipsychotic treatment responses for patients with schizophrenia, including immunological, neuroimaging and pharmacogenetic tests. However, the effect size of most stratifiers is small [10]. Recently, a large genome-wide association study conducted by the Chinese Antipsychotics Pharmacogenomics Consortium (CAPOC) showed that a combination of five novel loci could predict antipsychotic treatment response, with a sensitivity of $65 \%$ and a specificity of $69 \%$ [11]. It is therefore important to estimate whether using a test with this level of accuracy is sufficient to achieve cost-effective improvements in quality of life or not. This study aims to answer this question by estimating and comparing the long-term health and cost outcomes for patients who are treated with and without guidance of a hypothetical stratifier under different scenarios. The hypothetical stratifier can be any tests, such as immunological, neuroimaging or pharmacogenetic tests.

\section{Methods}

This study was reported according to the CHEERS recommendations for reporting health economic evaluations [12].

\section{Population}

The base case for the analysis consisted of a hypothetical cohort of adult patients with schizophrenia who failed a firstline conventional (i.e. non-clozapine) antipsychotic in the UK. It was decided that a stratifier would be more useful for patients who failed a first-line conventional antipsychotic than patients who are treatment-naïve, because the latter patient group has a high response rate to first-line conventional antipsychotic (ranges from 67.4 to $75.4 \%$ as reported by published literature $[13,14])$.

\section{Competing treatment strategies}

Two treatment strategies were considered in the model: treatment as usual (TAU) and a stratified medical algorithm

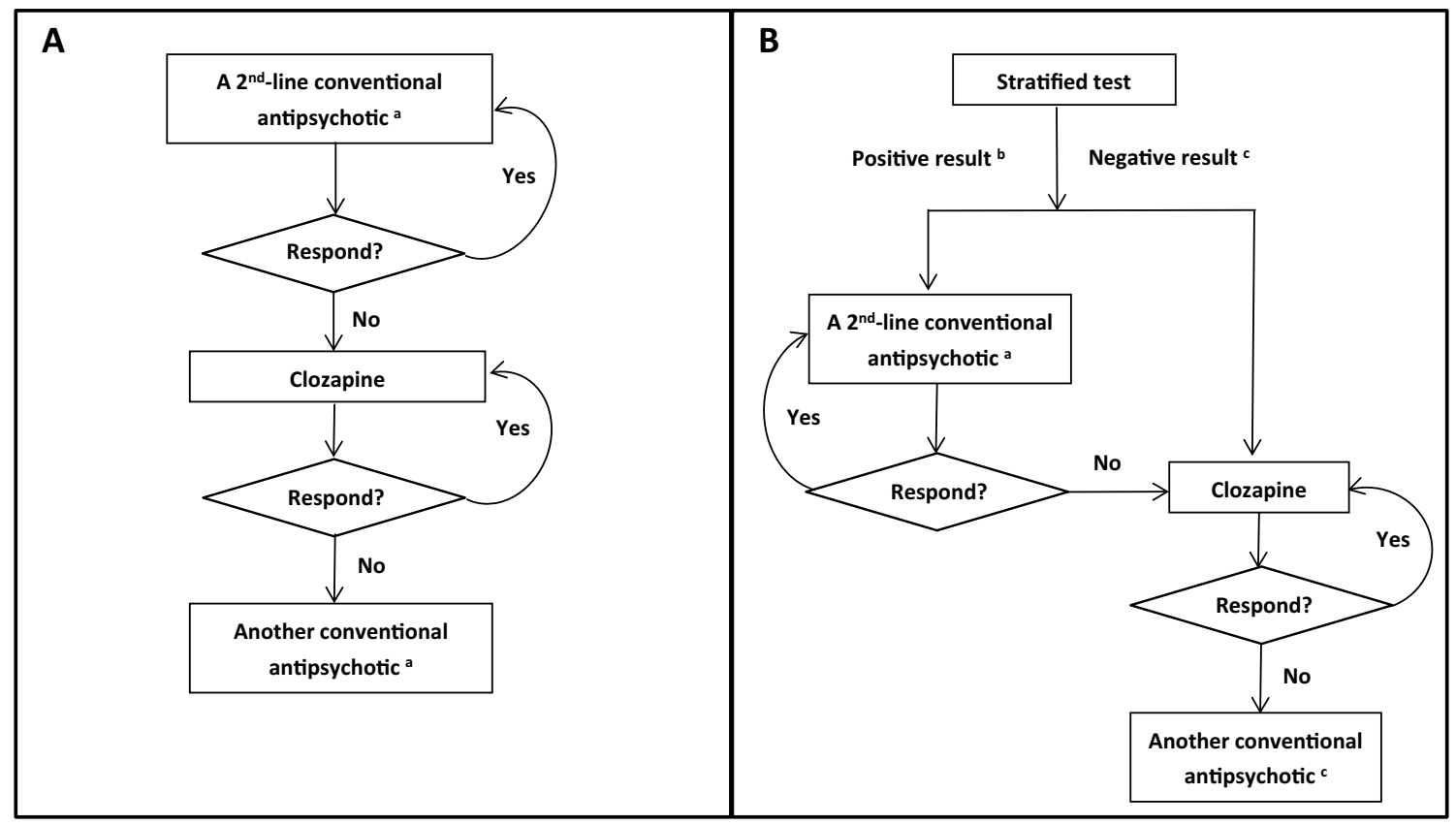

Fig. 1 Protocol of treatment as usual (TAU) and stratified medical algorithm (SMA) for schizophrenia patients who failed a first-line conventional antipsychotic. a The conventional antipsychotics considered in the model include: olanzapine, amisulpride, aripiprazole, paliperidone, risperidone, haloperidol and flupenthixol decanoate. b Predicted to respond to a second-line conventional antipsychotic. c Predicted not to respond to a second-line conventional antipsychotic 
(SMA). Both strategies are described briefly below, and are visually shown in Fig. 1a, b, respectively. The primary outcomes of this analysis are lifetime cost and quality-adjusted life year (QALYs) gained for patients who received one of the alternative treatment strategies (TAU or SMA).

\section{TAU}

Patients would be treated according to the National Institute for Health and Care Excellence (NICE) schizophrenia guideline 2014 [2]. Thus, all patients who failed a first-line conventional antipsychotic would be treated with a second-line conventional antipsychotic. For this study, the seven antipsychotics included in the network meta-analysis conducted by the NICE schizophrenia guideline were modelled as secondline conventional antipsychotics, including olanzapine, amisulpride, aripiprazole, paliperidone, risperidone, haloperidol and flupenthixol decanoate [2]. Patients who did not respond to a second-line antipsychotic would be switched to clozapine; while patients who did not respond to, or experienced an intolerable adverse reaction to clozapine, would be switched to another conventional antipsychotic.

\section{SMA}

All patients who failed a first-line conventional antipsychotic would undergo a stratified test designed to predict their response to second-line conventional antipsychotics. Patients predicted to be AP2 responders (positive test result) would be switched to a second-line conventional antipsychotic; while patients predicted not to be AP2 responders (negative test result) would be switched immediately to clozapine, thus bypassing the second treatment trial. Patients who failed a second-line conventional antipsychotic would be switched to clozapine; while patients who failed clozapine would be switched to another conventional antipsychotic.

\section{Key impacts of the stratifier modelled in the study}

In order to estimate the lifetime impact of the stratifier on total cost and QALYs, the following impacts of using a stratifier were modelled: clinical benefits, clinical harms, cost of stratifier, and cost impact on other resource use. A brief description of each impact is presented below. The clinical benefit of using SMA considered in the model is that clozapine responders who are correctly identified as AP2 non-responders (true negative) can initiate clozapine once they fail a first-line antipsychotic, without having to try an ineffective second-line antipsychotic first. Therefore, the costs saved from treating otherwise relapsed patients, the increase in QALYs arising from spending less time in a state of relapse, and their reduced mortality accruing from the use of clozapine, were modelled. The potential harm of using
SMA is, if the stratifier is not $100 \%$ accurate, some AP2 responders might be wrongly identified as AP2 non-responders (false negative) and switched to clozapine unnecessarily. AP2 responders who were wrongly predicted to be AP2 non-responders (false negatives), and were thus prescribed clozapine, would have a $71.16 \%$ probability of responding to clozapine and therefore remain on it $[13,15]$. The remaining $28.84 \%$ patients who do not respond to clozapine would be offered a second-line conventional antipsychotic, which they would respond to. Compared to most conventional antipsychotics included in the model, clozapine is associated with higher adherence rate and lower incidence of acute extrapyramidal symptoms (EPS), but is more likely to cause other adverse reactions, including weight gain and diabetes [2]. Furthermore, patients on clozapine are at risk of neutropenia and thus need to be monitored frequently. There is therefore a cost to being unnecessarily prescribed clozapine, which was included in the model.

\section{Model structure}

In order to capture the key impacts of using a stratifier (as described above), a decision analytic model with a Markov process embedded was developed using TreeAge 2014 (TreeAge Software, Williamstown, MA). Within the Markov process, events of interest are modelled as transitions from one health state to another. The time period of the model is divided into cycles of time, for example a year for this study, and at each cycle, probabilities are assigned of remaining in the same state or moving to a different state within the model. A 1-year cycle was chosen because most antipsychotic discontinuation happened within a year after the initiation of a new antipsychotic [16]. The economic model built for the NICE schizophrenia guideline also adopts a 1-year cycle [2]. In our model, eleven mutually exclusive health states were included. The transition between different health states for patients in the TAU arm and the SMA arm are illustrated in Figs. 2 and 3, respectively. Which health state the patient would be in depends on the patient's subgroup (AP2 responder, clozapine responder, or non-responder) and which antipsychotic treatment the patient is currently on (conventional antipsychotic, clozapine, or no antipsychotic due to non-adherence). Each of the health states is assigned a cost and effectiveness value that patients accrue while in that state. For each treatment, the overall costs and effectiveness are calculated on the basis of the total length of time patients spend in each health state over the time horizon. Both costs and QALYs were discounted at an annual rate of $3.5 \%$, as recommended by NICE [17], to reflect society's preference for costs to be incurred in the future rather than the present, and for benefits to be experienced in the present rather than the future. Use of antipsychotics are associated with a wide range of adverse physical and mental health events. Based on 


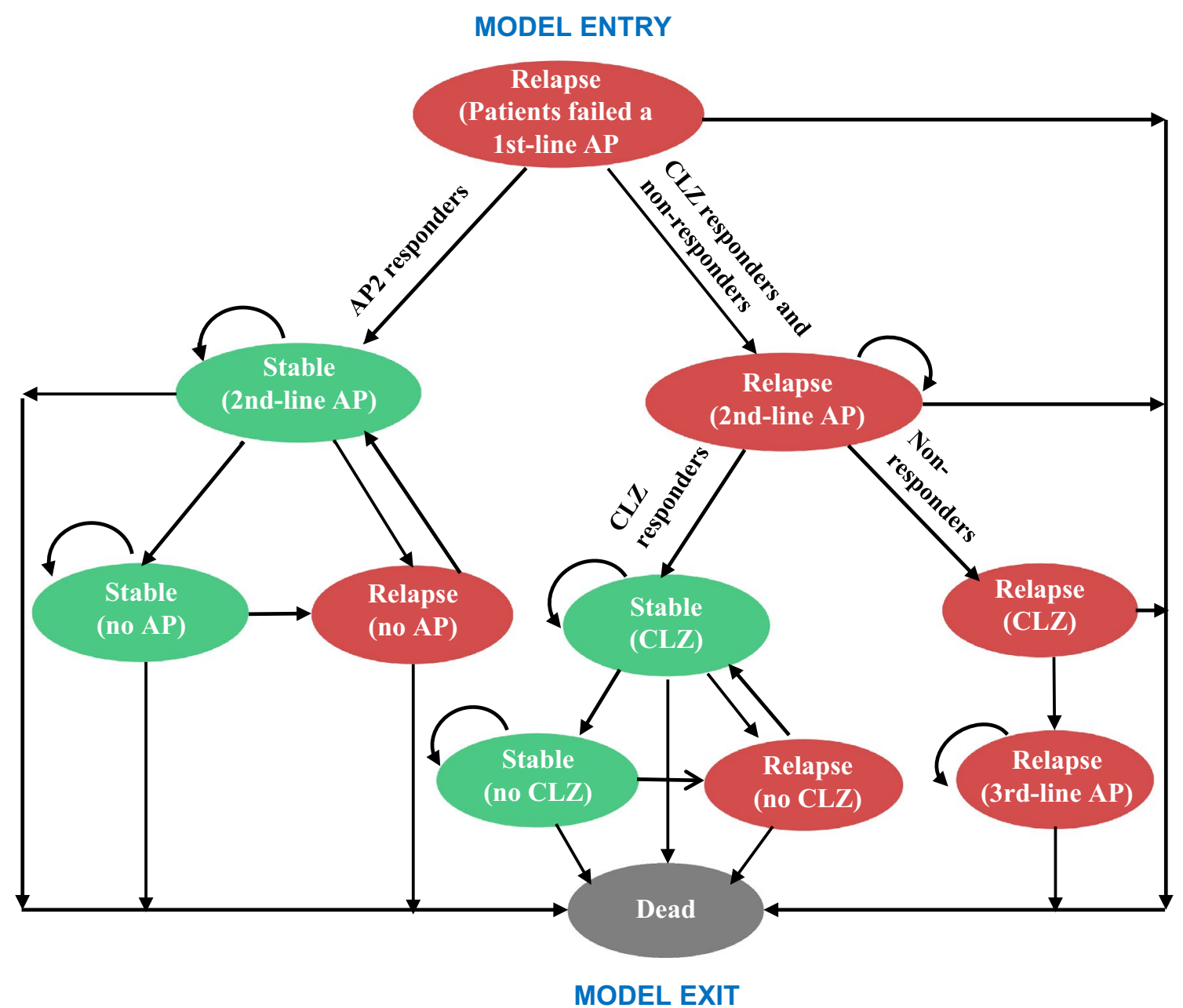

Fig. 2 State transition diagram of the Markov model for patients in the TAU arm. AP conventional antipsychotic, CLZ clozapine

the incidence rate, magnitude of health and cost impact and data availability, four adverse events of antipsychotic medications were selected for this model: weight gain, acute EPS, diabetes and neutropenia (for patients on clozapine only).

It was assumed that within the target population, there are three patient subgroups:

- AP2 responder, patients who will respond to a secondline conventional antipsychotic.

- Clozapine responder, patients who will fail to respond to a second line antipsychotic but will subsequently respond to clozapine.

- Non-responder, patients who will not respond to any existing antipsychotics including clozapine.

Wherever a patient responded to an antipsychotic, it was assumed that they would continue to receive the same antipsychotic in the following cycles. Patients who responded to a second-line antipsychotic but did not adhere to their medication were assumed to remain without antipsychotic treatment until they experienced a relapse, after which they were assumed to return to the same antipsychotic.

\section{Input data}

Cost-effectiveness analysis requires four types of data: clinical evidence, health-related preferences (utilities), healthcare resource use and costs. A summary of the key parameters used in the model is reported in Table 1 and briefly described below; while a detailed description of all parameters used in the model is reported in Online Resource 1. In the base-case analysis, both the sensitivity and specificity of the stratifier were set at $60 \%$ (similar to the accuracy data reported by the 


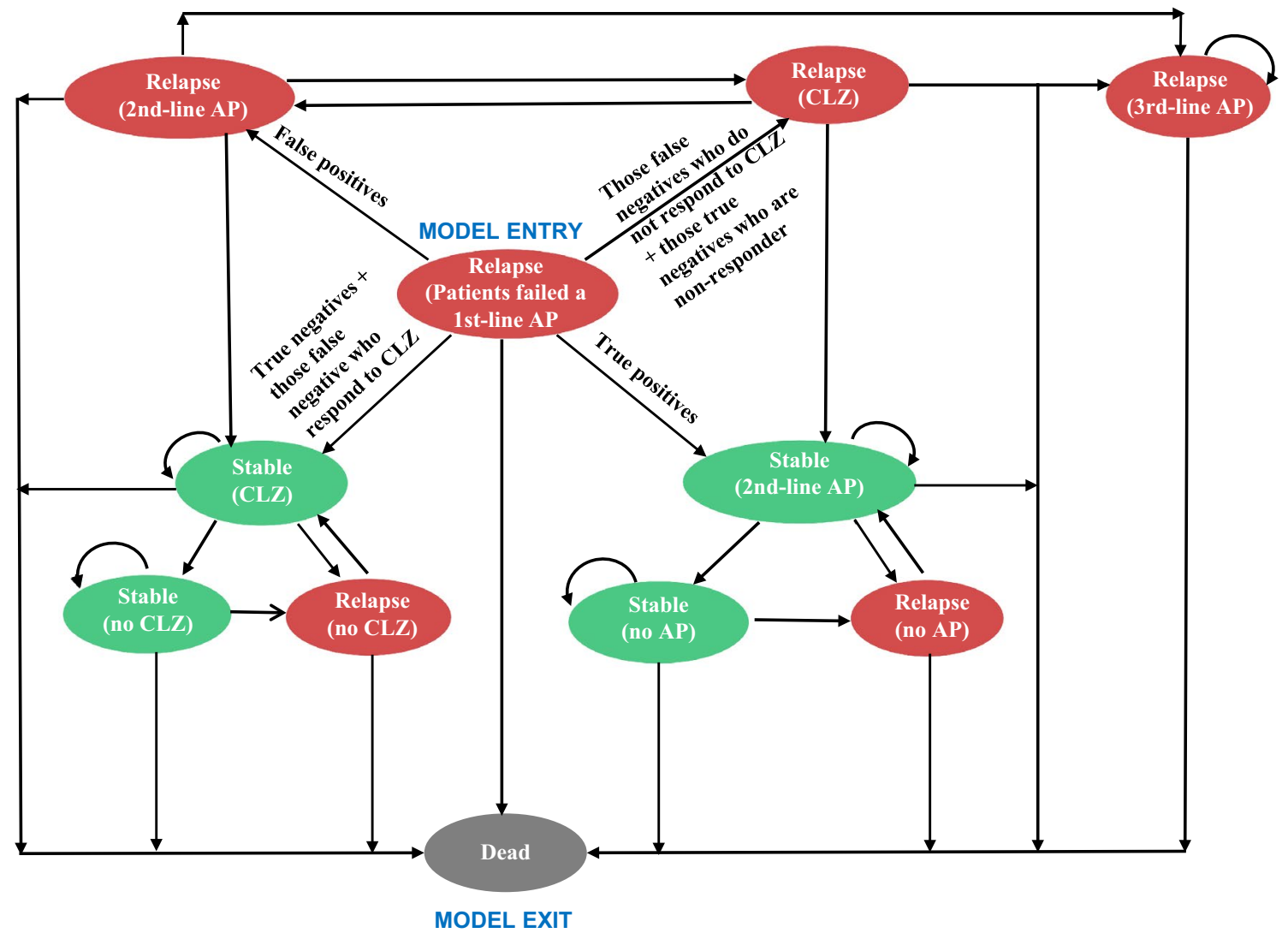

Fig. 3 State transition diagram of the Markov model for patients in the SMA arm. AP conventional antipsychotic, CLZ clozapine

study conducted by CAPOC [11]), with a range of $0-100 \%$ was tested in sensitivity analyses. There is a lack of clinical evidence about patients' response to a second-line antipsychotic after failing a first-line antipsychotic. According to a recent literature review [14], there are only two trials which examined patients' response to a second-line antipsychotic after failing a first-line antipsychotic: one is a naturalistic study conducted by Agid et al. [13], and another is an openlabel treatment with clozapine in first-episode schizophrenia and schizophreniform disorder (OPTiMiSE) trial [14]. It was decided that the data reported by Agid et al. is more appropriate for this model for two reasons: (1) Agid et al. has a larger sample size than the OPTiMiSE trial (60 vs 39, number of patients who were switched to a second-line antipsychotic after failing a first-line antipsychotic); (2) The results of the OPTiMiSE trial indicated that for patients who did not achieve remission with a first-line antipsychotic within 4 weeks, switching to a second-line conventional antipsychotic did not improve their response rate-or in another word, for patients who failed a first-line antipsychotic, the proportion of AP2 responder is nearly zero. The lower the proportion of AP2 responder, the higher the proportion of clozapine responder and non-responder, and the more costeffective the stratifier, as the key benefit of using the stratifier is to help clozapine responders to initiate clozapine earlier compared to the current practice. Therefore, to be conservative about the potential benefits of using the stratifier, the data reported by Agid et al. was used in the base case analysis, while a different range of data was tested in sensitivity analyses. Based on the data reported by Agid et al., it was calculated that for patients who failed a first-line antipsychotic, $16.67 \%$ of them responded to a conventional secondline antipsychotic (risperidone or olanzapine was used in the study), $62.50 \%$ responded to clozapine, and $20.83 \%$ did not respond to any antipsychotic tested in the study. Therefore, in the model, it was assumed that the proportion of different patient subgroups are: AP2 responder (16.67\%), clozapine responder $(62.50 \%)$ and non-responder $(20.83 \%)$. The proportion of positive and negative test results depend on the sensitivity and specificity of the stratifier, and the proportion of AP2 responders. Under a stratifier with $60 \%$ sensitivity and specificity, and assuming $16.67 \%$ of target population are AP2 responders, $43.33 \%$ patients will get positive results ( $10.00 \%$ are true positive and $33.33 \%$ are false positive), while $56.67 \%$ patients will get negative results $(50.00 \%$ are true negative and $6.67 \%$ are false negative). Patients' probability of non-adherence to conventional antipsychotics and probability of developing adverse events were obtained from 
Table 1 Summary of key parameters used in model

\begin{tabular}{|c|c|c|c|c|c|c|c|}
\hline Parameters & $\begin{array}{l}\text { Base-line } \\
\text { value }\end{array}$ & \multicolumn{2}{|c|}{$\begin{array}{l}\text { Range tested in one-way } \\
\text { or two-way sensitivity analysis }\end{array}$} & \multicolumn{3}{|l|}{ Distribution } & Source \\
\hline \multicolumn{8}{|c|}{ Diagnostic efficacy of predictive test } \\
\hline $\begin{array}{l}\text { Sensitivity } \\
\text { (proportion } \\
\text { of second- } \\
\text { line antip- } \\
\text { sychotic } \\
\text { responders } \\
\text { that are } \\
\text { correctly } \\
\text { identified } \\
\text { as such) }\end{array}$ & 0.60 & \multicolumn{2}{|l|}{$0-1.00$} & \multicolumn{3}{|l|}{ Assumed fixed } & $\begin{array}{l}\text { Estimate of what may } \\
\text { be achievable }\end{array}$ \\
\hline $\begin{array}{l}\text { Specificity } \\
\text { (propor- } \\
\text { tion of } \\
\text { second-line } \\
\text { antipsy- } \\
\text { chotic non- } \\
\text { responders } \\
\text { that are } \\
\text { correctly } \\
\text { identified } \\
\text { as such) }\end{array}$ & 0.60 & \multicolumn{2}{|l|}{$0-1.00$} & \multicolumn{3}{|l|}{ Assumed fixed } & $\begin{array}{l}\text { Estimate of what may } \\
\text { be achievable }\end{array}$ \\
\hline \multicolumn{8}{|c|}{ Distribution of patients who failed a first-line antipsychotic by subsequent response/non-response } \\
\hline $\begin{array}{l}\text { Clozapine } \\
\text { responder }\end{array}$ & $62.50 \%$ & \multicolumn{2}{|l|}{$0 \%-79.15 \%$} & \multicolumn{3}{|c|}{ Dirichlet distribution $(n=21.0)$} & Agid et al. [15] \\
\hline $\begin{array}{l}\mathrm{AP} 2 \\
\text { responder }\end{array}$ & $16.67 \%$ & \multicolumn{2}{|l|}{ N/A } & \multicolumn{3}{|c|}{ Dirichlet distribution $(n=5.6)$} & As above \\
\hline $\begin{array}{l}\text { Clozapine } \\
\text { non- } \\
\text { responder }\end{array}$ & $20.83 \%$ & \multicolumn{2}{|l|}{ N/A } & \multicolumn{3}{|c|}{ Dirichlet distribution $(n=7.0)$} & As above \\
\hline \multicolumn{8}{|c|}{ Response to different antipsychotics in misclassified individuals } \\
\hline $\begin{array}{l}\text { AP2 } \\
\text { responder's } \\
\text { response to } \\
\text { clozapine }\end{array}$ & $71.16 \%$ & \multicolumn{2}{|c|}{$0-1$} & \multicolumn{3}{|c|}{$\begin{array}{l}\text { Beta distribution (SD assumed to be } 50 \% \text { of } \\
\text { mean value) }\end{array}$} & $\begin{array}{l}\text { Calculated from Agid } \\
\text { et al. [15] and McE- } \\
\text { voy et al. [1] }\end{array}$ \\
\hline $\begin{array}{l}\text { AP2 non- } \\
\text { responder } \\
\text { response to } \\
\text { second-line } \\
\text { antipsy- } \\
\text { chotics }\end{array}$ & $0 \%$ & \multicolumn{2}{|l|}{ N/A } & \multicolumn{3}{|l|}{ Assumed fixed } & Estimate \\
\hline \multicolumn{8}{|l|}{ Cost data } \\
\hline \multicolumn{2}{|c|}{ Cost of predictive test } & \multicolumn{2}{|c|}{$£ 500.00$} & & $\begin{array}{l}£ 100.00- \\
1,000.00\end{array}$ & $\begin{array}{l}\text { Gamma distribu } \\
\text { assumed to be } \\
\text { value) }\end{array}$ & $\begin{array}{l}\text { tion }(\mathrm{SD} \\
50 \% \text { of mean }\end{array}$ \\
\hline \multicolumn{2}{|l|}{ Interventions } & Base-line value & $\begin{array}{l}\text { Range tes } \\
\text { two-way }\end{array}$ & $\begin{array}{l}\text { ed in one-way or } \\
\text { ensitivity analysis }\end{array}$ & Distribution & & Source \\
\hline \multicolumn{2}{|c|}{$\begin{array}{l}\text { Annual cost of treating patients } \\
\text { with active psychosis }\end{array}$} & $£ 39,141$ & N/A & & As above & & $\begin{array}{l}\text { Uplifted from the NICE } \\
\text { schizophrenia guide- } \\
\text { line [2] }\end{array}$ \\
\hline \multicolumn{2}{|c|}{$\begin{array}{l}\text { Annual cost of treating remitted } \\
\text { patients }\end{array}$} & $£ 15,086$ & $£ 10,000-$ & 39,141 & As above & & As above \\
\hline \multicolumn{8}{|c|}{ Health-related quality of life data } \\
\hline \multicolumn{2}{|l|}{ Relapse } & 0.4790 & $0.1900-0$ & 5040 & Beta distribut & ion (SD: 0.0330) & Briggs et al. [24] \\
\hline \multicolumn{2}{|c|}{ Stable schizophrenia } & 0.8650 & $0.8650-0$ & 9190 & Beta distribut & ion (SD: 0.0210) & As above \\
\hline
\end{tabular}


Table 1 (continued)

\begin{tabular}{llllc}
\hline Interventions & Base-line value & $\begin{array}{l}\text { Range tested in one-way or } \\
\text { two-way sensitivity analysis }\end{array}$ & Distribution & Source \\
\hline $\begin{array}{l}\text { Other input data } \\
\begin{array}{l}\text { Annual discount rate for both } \\
\text { costs and outcomes }\end{array}\end{array}$ & 0.0350 & $0-0.050$ & Assumed fixed & NICE guideline manual \\
$\begin{array}{l}\text { Cycle length } \\
\text { Number of cycles }\end{array}$ & $\begin{array}{l}1 \text { year } \\
\text { Num }\end{array}$ & N/A & Assumed fixed & Estimate \\
\hline
\end{tabular}

A complete list of all parameters used in the model is reported in Online Resource 2, Table 1

Table 2 Verification and validation process and people involved

\begin{tabular}{ll}
\hline Verification and validation process & People involved \\
\hline 1. Check appropriateness of the model structure & JM and PM \\
2. Check appropriateness of data source & JM and PM \\
3. Compare data used in the model against the evidence sources & HJ \\
4. Check model logic (white-box testing) & HJ \\
5. Check the plausibility of the intermediate and final outputs, including & HJ, JM and PM \\
results of sensitivity analyses (black-box testing) & \\
6. Compare results with published literature & HJ
\end{tabular}

$H J$ Huajie Jin, $J M$ James MacCabe, $P M$ Paul McCrone the network meta-analyses reported in the NICE schizophrenia guideline [2].

Utility data were obtained from a UK study which reported separate utility scores for stable or relapsed schizophrenia patients with or without adverse events of antipsychotics [18]. For costing data, the model took the perspective of the UK health and social care system, as recommended by NICE [17]. All costs were reported in 2017 UK pounds. The cost of the stratifier was assumed to be $£ 500$ per patient (an estimate of the cost of a magnetic resonance spectroscopy scan, which is considered to be the higher end of the likely cost of a stratifier) with a range of $£ 100-1000$ tested in sensitivity analyses [19]. Unit costs were based on the NHS reference costs 2016-17 [20], prescription cost analysis (England) 2017 [21] or the Unit Costs of Health and Social Care 2017 [22]. Resource quantities were mainly informed by the NICE schizophrenia guideline 2014 [2].

\section{Sensitivity analysis}

The credibility of the results of economic analysis largely relies on the validity of input data. Sensitivity analyses can test the effect of changes in the input data on the observed results. If, after performing sensitivity analyses, the findings are consistent with those from the baseline analysis and would lead to similar conclusions about the cost-effectiveness of different strategies, we can be reassured that the
Table 3 Incremental costs and QALYs per person by treatment strategy

\begin{tabular}{lllll}
\hline Strategy & Total Costs $(\mathfrak{E})$ & Total QALYs & $\begin{array}{l}\text { Incremen- } \\
\text { tal cost }(\mathfrak{f})\end{array}$ & $\begin{array}{l}\text { Incre- } \\
\text { mental } \\
\text { QALYs }\end{array}$ \\
\hline TAU & 510,239 & 16.15 & - & - \\
SMA & 502,876 & 16.25 & -7363 & 0.10 \\
\hline
\end{tabular}

uncertainty of input data has little impact on the primary conclusions. For this study, three types of sensitivity analyses were conducted: one-way sensitivity analysis which assess the impact of uncertainty around the value of each individual parameter, two-way sensitivity analysis which assess the impact of uncertainty around the value of two correlated parameters (e.g. sensitivity and specificity of the stratifier), and probabilistic sensitivity analysis (PSA) which examine the impact of joint uncertainty of multiple parameters simultaneously. A summary of all parameters tested in sensitivity analysis is reported in Online Resource 1 , Table 1.

\section{Model verification and validation}

A summary of the activities conducted for model verification and validation is presented in Table 2 . 


\section{Results}

The base-case analysis results are presented in Table 3 . Compared to TAU, use of the SMA produced 0.10 more QALYs and incurred $£ 7363$ lower costs per person. The results of one-way and two-way sensitivity analysis are reported in detail in Online Resource 2 and is summarised below. One-way sensitivity analysis showed that our conclusion (SMA is more cost-effective than TAU) was robust to all parameters tested. Two-way sensitivity analysis shows that even when the sensitivity of the stratifier is $0 \%$, as long as the specificity of the stratifier is no less than $11.50 \%$, SMA is still more cost-effective than TAU. If the sensitivity of the stratifier is 50\%, as long as the specificity of the test is no less than $6 \%$, SMA is more cost-effective than TAU. The result of PSA is shown in Fig. 4. If the NHS was not willing to attach any monetary value to QALY gains, the likelihood for SMA to be the most cost-effective option is $76.62 \%$. As a unit improvement is valued at higher levels this likelihood increases. At a WTP threshold of $£ 20,000$ per QALY, there is a $83.04 \%$ likelihood that SMA is the most cost-effective option.

\section{Discussion}

\section{Main findings and interpretation}

Significant efforts have been made to identify stratifiers for predicting antipsychotic treatment responses for patients with schizophrenia. However, little is known about how accurate such a stratifier needs to be to achieve cost-effective improvements in quality of life. To the best of our knowledge, only one previous study has assessed the cost-effectiveness of a stratifier for schizophrenia patients, which is a modelling study conducted by Perlis et al. [23]. The stratifier assessed was a genetic test that can identify individuals with greater likelihood of responding to second-line conventional antipsychotics, with a sensitivity of $95.9 \%$ and a specificity of $38.3 \%$. Perlis et al. found that applying the test to treatment-naïve patients and using clozapine as a first-line antipsychotic for those predicted to respond to clozapine is more effective but also more expensive, comparing with no test and reversing clozapine as a third-line antipsychotic. However, the study conducted by Perlis et al. had some limitations: (1) it did not consider the health or cost impact of any adverse events of antipsychotics, which might have important impacts on the cost-effectives results; (2) PSA was not conducted to examine the impact of joint uncertainty of multiple parameters simultaneously; (3) this study was
Fig. 4 Cost-effectiveness acceptability curves showing probability that each strategy is most cost-effective option at different willingness-to-pay (WTP). SMA Stratified medicine algorithm. TAU Treatment as usual

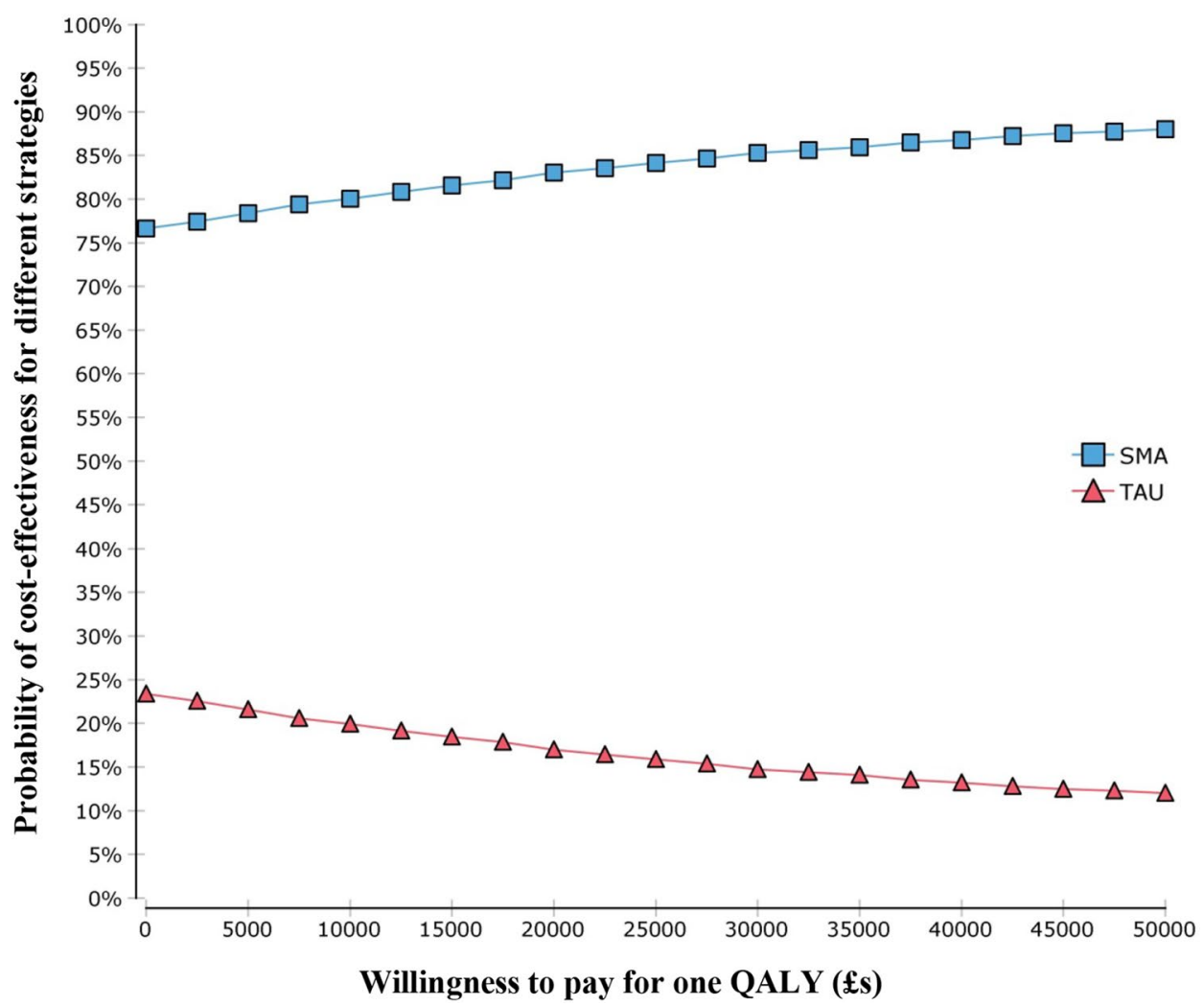


published in 2005, therefore, it has limited relevance to the current context, due to rapidly changing nature of treatments, health services, evidence base and unit costs. No previous studies have assessed the cost-effectiveness of a stratifier for schizophrenia patients who fail a first-line antipsychotic. In order to fill the gap, we used decision-analytic modelling to estimate the cost-effectiveness of a SMA for schizophrenia patients who failed a first-line antipsychotic, over a life-time time horizon from the health sector and social care perspective. Results indicate that the stratified medicine algorithm was more cost effective that treatment as usual, when both the sensitivity and specificity of the stratifier was set at $60 \%$. One surprising finding of this study is, even when the sensitivity of the stratifier is set to $0 \%$, as long as the specificity of the stratifier is no less than $11.50 \%$, SMA is still more costeffective than TAU. This was mainly because the proportion of patients who would respond to clozapine is much larger than the proportion of patients who would only respond to a second-line conventional antipsychotic. A $0 \%$ sensitivity means none of the AP2 responders are correctly identified as such-all of them are identified as AP2 non-responders and therefore be prescribed with clozapine. According to Agid's study [13] and the CATIE trial [15], 16.67\% of the target population are AP2 responders; and $71.16 \%$ of AP2 responders would also respond to clozapine. This means, only those AP2 responders who do not respond to clozapine $(16.67 \% *(1-71.16 \%)=4.81 \%)$ are adversely affected by the poor sensitivity of the stratifier, and even then, they are only on clozapine for 1 year in our model, before being switched to a non-clozapine antipsychotic, to which they would respond. For those AP2 responder who also respond to clozapine $(16.67 \% * 71.16 \%=11.86 \%)$, our model shows that although they are slightly better-off with a conventional second-line antipsychotic comparing to clozapine, the overall magnitude of cost and QALY difference is very small. The specificity of the test, in this model, is its ability in correctly identifying AP2 non-responders, which includes clozapine responders and non-responders. According to Agid's study [13], clozapine responders comprise $62.50 \%$ of the target population. A $11.50 \%$ specificity means, comparing to TAU, $7.19 \%(=11.50 \% * 62.50 \%)$ of the target population received the right treatment (clozapine) one cycle earlier, and therefore results in lower cost and higher QALY gains. To sum up, comparing to TAU, use of a stratifier with $0 \%$ sensitivity and $11.50 \%$ specificity results in worse outcomes for $4.81 \%$ patients (AP2 responders wrongly identified as clozapine responders, and who do not respond to clozapine) and better outcomes for $7.19 \%$ patients (clozapine responders correctly identified as AP2 non-responders). Therefore, after weighing up the clinical benefits, clinical harms and cost implications of the stratifier, SMA is considered to be more cost-effective than TAU.
According to a recent systematic review [14], there are only two trials which examined patients' response to a second-line antipsychotic after failing a first-line antipsychotic: Agid et al. [13] and the OPTiMiSE trial [14]. Both studies found that for patients who failed a first-line conventional antipsychotic, their response to a second-line conventional antipsychotic is poor-in fact, the OPTiMiSE trial found that for patients who did not achieve remission with a firstline antipsychotic within 4 weeks, it might be better for them to continue their current antipsychotic, rather than being switched to a second-line conventional antipsychotic. The findings of these two studies, combined with the result of our model (a stratifier with very low sensitivity and specificity is still considered to be cost-effective) raises the question of whether a stratifier is necessary for patients who failed a first-line antipsychotic or not-using clozapine as a secondline treatment without any stratifier might turn out to be more cost-effective, compared to use of a stratifier with low sensitivity and/or specificity. Although a few studies have argued the potential benefits of using clozapine as a secondline or even first-line treatment [24-26], there is currently a lack of high-quality evidence about clozapine's efficacy for schizophrenia who failed a first-line antipsychotic. Further research is urgently needed to establish clozapine's clinical efficacy and safety profile for patients as a second-line treatment. With publication of such results, the input data of the present analysis can be updated to shed light on whether clozapine should be used as a second-line antipsychotic.

\section{Strengths and limitations}

To our knowledge, this is the first study to explore the pharmacoeconomic implications of using a stratified medicine approach in schizophrenia patients who failed a first-line antipsychotic. A major strength of this study was the collective use of the state-of-the-art methodology and most up-to-date data to evaluate the cost-effectiveness of a hypothetical stratifier. Decision analytic modelling provides a framework to quantify and synthesize the clinical benefits, clinical harms and cost implications of the stratifier. Comprehensive sensitivity analyses have been conducted to test the uncertainty of the result. Therefore, commissioners and clinical researchers can be reassured that the conclusion of this paper is robust to large changes or errors in the input parameters.

There are a number of limitations of the economic model presented here, the majority of which derive from limitations in the evidence base used to populate the model. For example: (1) patients' responses to second-line antipsychotics were calculated based on the data for 60 schizophrenia patients included in the Agid et al. [13], as there is a lack of larger trials which specifically assessed patients' responses to a second-line antipsychotic after failing a first-line 
antipsychotic. There is uncertainty about the generalizability of the results reported by Agid et al. due to its small sample size; (2) patients' probability of developing adverse events were obtained from the network meta-analyses reported in the NICE schizophrenia guideline [2]. It was noted that the population included in those network meta-analyses were 'general' schizophrenia patients in remission, some of whom might have been on antipsychotics for many years. It is unknow whether those patients would have different probability of developing adverse events, compared with our target population. However, in this study, the limitation related to parameter uncertainty has been partially mitigated by extensive sensitivity analyses. Secondly, discontinuation of antipsychotics due to intolerable adverse events was only modelled for clozapine, but not for other conventional antipsychotics. This simplification is against the use of clozapine, and thus against the use of stratifier, as the key benefit of using the stratifier is that clozapine responders who are correctly identified as AP2 non-responders can initiate clozapine sooner than the current practice. The base case results show that even when this simplification was used, SMA still dominates TAU, which demonstrates the robustness of our base case conclusion. Thirdly, the cost and health impacts of adverse events of antipsychotics have not been fully captured. Other than the four adverse events modelled in this study (i.e. weight gain, diabetes, acute EPS and neutropenia), there are other adverse events which might impact on patients' QALY and cost outcomes, such as tardive dyskinesia, sexual dysfunction, increase in prolactin levels, and agranulocytosis. Even for those adverse events which were considered in the model, the impacts of complications of those adverse events were not fully captured: for example, the impact of complications of diabetes (e.g. myocardial infarction and stroke etc.) on mortality were not considered in the model. Fourthly, besides the health and social care costs that were considered in this analysis, SMA is likely to have an impact on the wider societal costs (such as costs borne to the criminal justice system and productivity losses for schizophrenia patients and their carers). When wider societal costs were considered, the gap between treatment cost of remitted patients and relapse patients is likely to widen [27], and thus making SMA more cost-effective.

\section{Conclusion}

Our results suggest that use of any stratifier (e.g. immunological, neuroimaging or pharmacogenetic tests) with a sensitivity and specificity over $60 \%$ is very likely to be cost-effective comparing to TAU, for psychotic patients who failed a first-line antipsychotic. This finding, however, should be interpreted with caution due to lack of evidence for clozapine as a second-line antipsychotic.

Acknowledgements The authors thank Dr. Carol Paton for estimating the resource use of patients' visit to a clozapine clinic.

Author contributions The model was specified in close collaboration between Huajie Jin (health economist who specified the methodology, built the model, and defined the parameters), Paul McCrone (health economist who advised on and validated the model and helped interpret the results) and James MacCabe (clinical expert who guided the definition of strategies, advised on model structure and parameters). Huajie Jin wrote the first draft of the paper, which was subsequently been edited by all authors who have approved the final version. Huajie Jin will serve as a guarantor for the overall content of the manuscript.

Funding This work was funded by the Medical Research Council, UK, Grant number MR/L011794/1. This study represents independent research part funded by the National Institute for Health Research (NIHR) Biomedical Research Centre at South London and Maudsley NHS Foundation Trust and King's College London. The sponsor and funder had no role in the design, data collection, analysis, interpretation, writing, or publication of this study. The views expressed are those of the authors and not necessarily those of the NHS, the NIHR or the Department of Health.

Data availability All data generated or analysed during this study are included in this published article and its electronical supplementary martial (Online Resource 1-2).

\section{Compliance with ethical standards}

Conflicts of interest Huajie Jin, Paul McCrone and James MacCabe have no conflicts of interest that are directly relevant to the contents of this study.

Open Access This article is distributed under the terms of the Creative Commons Attribution 4.0 International License (http://creativeco mmons.org/licenses/by/4.0/), which permits unrestricted use, distribution, and reproduction in any medium, provided you give appropriate credit to the original author(s) and the source, provide a link to the Creative Commons license, and indicate if changes were made.

\section{References}

1. Lally, J., MacCabe, J.H.: Personalised approaches to pharmacotherapy for schizophrenia. Adv. Psychiatric Treat. 22(2), 78-86 (2016). (Date of Publication: March 2016.;2016)

2. National Collaborating Centre for Mental Health. Psychosis and schizophrenia in adults. NICE guideline (CG178). 2014

3. Wimberley, T., MacCabe, J.H., Laursen, T.M., Sorensen, H.J., Astrup, A., Horsdal, H.T., et al.: Mortality and self-harm in association with clozapine in treatment-resistant schizophrenia. Am. J. Psychiatry 174(10), 990-998 (2017). https://doi.org/10.1176/ appi.ajp.2017.16091097

4. Galletly, C., Castle, D., Dark, F., Humberstone, V., Jablensky, A., Killackey, E., et al.: Royal Australian and New Zealand college of psychiatrists clinical practice guidelines for the management of schizophrenia and related disorders. Aust. N. Z. J. Psychiatry 50(5), 410-472 (2016). https://doi.org/10.1177/00048 67416641195 
5. Loosbrock, D.L., Zhao, Z., Johnstone, B.M., Morris, L.S.: Antipsychotic medication use patterns and associated costs of care for individuals with schizophrenia. J. Ment. Health Policy Econ. 6(2), 67-75 (2003)

6. Pringsheim, T., Addington, D.: Canadian schizophrenia guidelines: introduction and guideline development process. Can. J Psychiatry Rev. Can. Psychiatrie 62(9), 586-593 (2017). https:// doi.org/10.1177/0706743717719897

7. Howes, O.D., Vergunst, F., Gee, S., McGuire, P., Kapur, S., Taylor, D.: Adherence to treatment guidelines in clinical practice: study of antipsychotic treatment prior to clozapine initiation. Br J Psychiatry. 201(6), 481-485 (2012). (Date of Publication: December 2012.; 2012)

8. Hui, C.L.M., Honer, W.G., Lee, E.H.M., Chang, W.C., Chan, S.K.W., Chen, E.S.M., et al.: Long-term effects of discontinuation from antipsychotic maintenance following first-episode schizophrenia and related disorders: a 10 year follow-up of a randomised, double-blind trial. Lancet Psychiatry. (2018). https://doi. org/10.1016/s2215-0366(18)30090-7

9. Yoshimura, B., Yada, Y., So, R., Takaki, M., Yamada, N.: The critical treatment window of clozapine in treatment-resistant schizophrenia: secondary analysis of an observational study. Psychiatry Res. 250, 65-70 (2017). https://doi.org/10.1016/j.psych res.2017.01.064

10. Fond, G., d'Albis, M.-A., Jamain, S., Tamouza, R., Arango, C., Fleischhacker, W.W., et al.: The promise of biological markers for treatment response in first-episode psychosis: a systematic review. Schizophr. Bull. 41(3), 559-573 (2015). https://doi.org/10.1093/ schbul/sbv002

11. Yu, H., Yan, H., Wang, L., Li, J., Tan, L., Deng, W., et al.: Five novel loci associated with antipsychotic treatment response in patients with schizophrenia: a genome-wide association study. Lancet Psychiatry. 5(4), 327-338 (2018). https://doi.org/10.1016/ s2215-0366(18)30049-x

12. Husereau, D., Drummond, M., Petrou, S., Carswell, C., Moher, D., Greenberg, D., et al.: Consolidated health economic evaluation reporting standards (CHEERS) Statement. Pharmaco Econ. 31(5), 361-367 (2013). https://doi.org/10.1007/s40273-013-0032-y

13. Agid, O., Arenovich, T., Sajeev, G., Zipursky, R.B., Kapur, S., Foussias, G., et al.: An algorithm-based approach to first-episode schizophrenia: response rates over 3 prospective antipsychotic trials with a retrospective data analysis. J Clin Psychiatry. 72(11), 1439-1444 (2011). (Date of Publication: November 2011.; 2011)

14. Kahn, R.S., Winter van Rossum, I., Leucht, S., McGuire, P., Lewis, S.W., Leboyer, M., et al.: Amisulpride and olanzapine followed by open-label treatment with clozapine in first-episode schizophrenia and schizophreniform disorder (OPTiMiSE): a three-phase switching study. Lancet Psychiatry. 10, 11 (2018). https://doi.org/10.1016/s2215-0366(18)30252-9

15. McEvoy, J.P., Lieberman, J.A., Stroup, T.S., Davis, S.M., Meltzer, H.Y., Rosenheck, R.A., et al.: Effectiveness of clozapine versus olanzapine, quetiapine, and risperidone in patients with chronic schizophrenia who did not respond to prior atypical antipsychotic treatment. Am J Psychiatry. 163(4), 600-610 (2006). https://doi. org/10.1176/appi.ajp.163.4.600

16. Lieberman, J.A., Stroup, T.S., McEvoy, J.P., Swartz, M.S., Rosenheck, R.A., Perkins, D.O., et al.: Effectiveness of antipsychotic drugs in patients with chronic schizophrenia. N. Engl. J. Med. 353(12), 1209-1223 (2005). https://doi.org/10.1056/NEJMoa0516 88

17. National Institute for Health and Care Excellence. Developing NICE guidelines: the manual. 2014

18. Briggs, A., Wild, D., Lees, M., Reaney, M., Dursun, S., et al.: Impact of schizophrenia and schizophrenia treatment-related adverse events on quality of life: direct utility elicitation. Health Qual Life Outcomes 6, 105 (2008)

19. Mouchlianitis, E., Bloomfield, M.A.P., Law, V., Beck, K., Selvaraj, S., Rasquinha, N., et al.: Treatment-resistant schizophrenia patients show elevated anterior cingulate cortex glutamate compared to treatment-responsive. Schizophr. Bull. 42(3), 744-752 (2016). https://doi.org/10.1093/schbul/sbv151

20. Department of Health: NHS reference costs 2016 to 2017. Department of Health, UK, London (2017)

21. NHS Digital (2017). Prescription Cost Analysis-England 2016. Retrieved 3rd March 2016, from https://digital.nhs.uk/data-andinformation/publications/statistical/prescription-cost-analysis/ prescription-cost-analysis-england-2016

22. Curtis, L.: Unit Costs of Health \& Social Care 2017. Personal Social Services Research Unit, University of Kent, Canterbury (2017)

23. Perlis, R.H., Ganz, D.A., Avorn, J., Schneeweiss, S., Glynn, R.J., Smoller, J.W., et al.: Pharmacogenetic testing in the clinical management of schizophrenia: a decision-analytic model. J. Clin. Psychopharmacol. 25(5), 427-434 (2005)

24. Remington, Gary, Agid, Ofer, Foussias, George, Hahn, Margaret, Rao, Naren, Sinyor, Mark: Clozapine's role in the treatment of first-episode schizophrenia. Am. J. Psychiatry 170(2), 146-151 (2013). https://doi.org/10.1176/appi.ajp.2012.12060778

25. Vera, I., Rezende, L., Molina, V., Sanz-Fuentenebro, J.: Clozapine as treatment of first choice in first psychotic episodes. What do we know? Actas Espanolas de Psiquiatria. 40(5), 281-289 (2012)

26. Green, A.I., Schildkraut, J.J.: Should clozapine be a first-line treatment for schizophrenia? The rationale for a double-blind clinical trial in first-episode patients. Harv. Rev. Psychiatry 3(1), 1-9 (1995)

27. Jin, H., Mosweu, I.: The societal cost of schizophrenia: a systematic review. PharmacoEconomics. 35(1), 25-42 (2017). https:// doi.org/10.1007/s40273-016-0444-6

Publisher's Note Springer Nature remains neutral with regard to jurisdictional claims in published maps and institutional affiliations. 